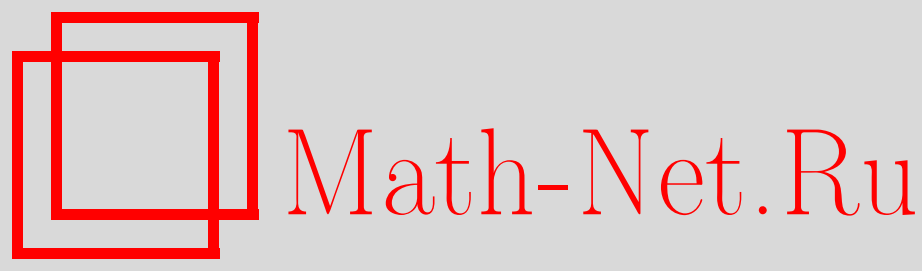

М. М. Гончаровский, И. В. Широков, Интегрируемый класс дифференциальных уравнений с нелокальной нелинейностью на группах Ли, ТMФ, 2009, том 161, номер 3, 332-345

DOI: https://doi.org/10.4213/tmf6445

Использование Общероссийского математического портала Math-Net.Ru подразумевает, что вы прочитали и согласны с пользовательским соглашением http: //www.mathnet.ru/rus/agreement

Параметры загрузки:

IP : 54.198 .64 .247

26 апреля 2023 г., 12:37:04

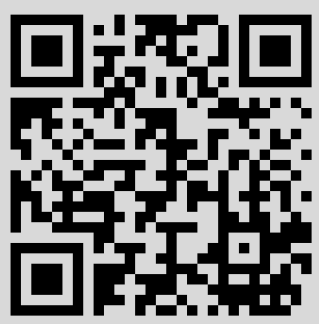




\title{
ИНТЕГРИРУЕМЫЙ КЛАСС ДИФФЕРЕНЦИАЛЬНЫХ УРАВНЕНИЙ С НЕЛОКАЛЬНОЙ НЕЛИНЕЙНОСТЬЮ НА ГРУППАХ ЛИ
}

\begin{abstract}
Построены общее и $N$-солитонное решения интегродифференциального уравнения Шредингера с нелокальной нелинейностью. Рассматриваются интегрируемые нелинейные интегродифференциальные уравнения на многообразии произвольной связной унимодулярной группы Ли. Для редукции уравнений на группе к уравнениям с меньшим числом независимых переменных применяется метод орбит коприсоединенного представления и построенный на его основе обобщенный гармонический анализ. Возможности изложенного алгоритма демонстрируются на примере группы $S O(3)$.
\end{abstract}

Ключевые слова: нелинейные интегродифференциальные уравнения, солитоны, группы Ли, коприсоединенное представление, гармонический анализ.

\section{1. ВВЕДЕНИЕ}

Актуальной задачей современной математической физики является задача построения интегрируемых нелинейных дифференциальных уравнений. В настоящей работе обсуждается класс интегрируемых уравнений с нелокальной нелинейностью на многообразиях групп Ли. В первой части работы рассматриваются интегрируемые нелинейные интегродифференциальные уравнения в пространстве $R^{n}$. Под интегрируемостью в этом случае понимается возможность сведения исходного уравнения к системе алгебраических или обыкновенных дифференциальных уравнений. Подробно рассматривается случай свободного уравнения Шредингера с дополнительным нелинейным интегральным членом, находится его общее решение, строится $N$-солитонное решение. Интересно, что, в отличие от классических нелинейных интегрируемых дифференциальных уравнений, допускающих солитонные решения (уравнение Кортевега-де Фриза (КдФ), нелинейное уравнение Шредингера (НУШ), синус-Гордон и т.д.), обобщение которых на многомерный случай представляет собой нетривиальную задачу (см., например, книгу [1]), нелокальные уравнения допускают многосолитонные решения в пространствах $R^{n}$ произвольной размерности.

* Омский государственный технический университет, Омск, Россия.

E-mail: iv_shirokov@mail.ru, eiklm@mail.ru 
Во второй части работы рассматривается более общий случай интегрируемых интегродифференциальных уравнений на многообразии $R^{1} \times G$, где $G$ - произвольная связная унимодулярная группа Ли. Для редукции уравнений на группе к уравнениям с меньшим числом независимых переменных применяется метод орбит коприсоединенного представления [2] и построенный на его основе в монографии [3] обобщенный гармонический анализ, ранее успешно применявшийся для интегрирования линейных дифференциальных уравнений [4], [5]. После редукции нелинейное интегродифференциальное уравнение сводится к системе алгебраических и обыкновенных дифференциальных уравнений. В качестве примера строится решение уравнения рассматриваемого типа для $G=S O(3)$.

\section{2. НЕЛИНЕЙНЫЕ ИНТЕГРОДИФФЕРЕНЦИАЛЬНЫЕ УРАВНЕНИЯ НА АБЕЛЕВОЙ ГРУППЕ}

В этом разделе $G$ - вещественная абелева $n$-мерная группа Ли, $\mathbf{x}=\left(x^{1}, \ldots, x^{n}\right)-$ координаты на многообразии $G$. Рассмотрим класс нелинейных интегродифференциальных уравнений для неизвестной функции $\psi(t, \mathbf{x}), t \in R^{1}$, следующего вида:

$$
\begin{aligned}
H\left(\partial_{t},\right. & \left.-i \partial_{x}\right) \psi(t, \mathbf{x})=A\left(\partial_{t},-i \partial_{x}\right) \int_{G^{3}} \psi\left(t, \mathbf{x}_{1}\right) \psi\left(t, \mathbf{x}_{2}\right) \overline{\psi\left(t, \mathbf{x}_{3}\right)} \times \\
& \times \delta\left(\mathbf{x}-\mathbf{x}_{1}-\mathbf{x}_{2}+\mathbf{x}_{3}\right) d \mathbf{x}_{1} d \mathbf{x}_{2} d \mathbf{x}_{3}+ \\
& +B\left(\partial_{t},-i \partial_{x}\right) \int_{G^{5}} \psi\left(t, \mathbf{x}_{1}\right) \psi\left(t, \mathbf{x}_{2}\right) \psi\left(t, \mathbf{x}_{3}\right) \overline{\psi\left(t, \mathbf{x}_{4}\right)} \overline{\psi\left(t, \mathbf{x}_{5}\right)} \times \\
& \times \delta\left(\mathbf{x}-\mathbf{x}_{1}-\mathbf{x}_{2}-\mathbf{x}_{3}+\mathbf{x}_{4}+\mathbf{x}_{5}\right) d \mathbf{x}_{1} d \mathbf{x}_{2} d \mathbf{x}_{3} d \mathbf{x}_{4} d \mathbf{x}_{5}+\cdots
\end{aligned}
$$

Здесь $H, A, B, \ldots$ - линейные дифференциальные операторы с постоянными коэффициентами. Введем преобразование Фурье $\psi(t, \mathbf{x})=\int e^{i \mathbf{p x}} \psi(t, \mathbf{p}) d \mathbf{p} /(2 \pi)^{n}$. Если какая-либо однопараметрическая подгруппа с координатой $x^{j}$ компактна: $x^{j} \sim$ $x^{j}+2 \pi$, то $p_{j} \in \mathbb{Z}$, и в формуле для преобразования Фурье интегрирование по переменной $p_{j}$ следует заменить суммированием по целым числам. После преобразования Фурье уравнение (1) переходит в обыкновенное дифференциальное уравнение

$$
H\left(\partial_{t}, \mathbf{p}\right) \psi(t, \mathbf{p})=A\left(\partial_{t}, \mathbf{p}\right)|\psi(t, \mathbf{p})|^{2} \psi(t, \mathbf{p})+B\left(\partial_{t}, \mathbf{p}\right)|\psi(t, \mathbf{p})|^{4} \psi(t, \mathbf{p})+\cdots
$$

Популярным объектом исследования является НУШ и его различные обобщения. Рассмотрим уравнение

$$
i \frac{\partial \psi(t, \mathbf{x})}{\partial t}+\Delta \psi(t, \mathbf{x})=\int K\left(\mathbf{x}, \mathbf{x}_{1}, \mathbf{x}_{2}, \mathbf{x}_{3}\right) \psi\left(t, \mathbf{x}_{1}\right) \psi\left(t, \mathbf{x}_{2}\right) \overline{\psi\left(t, \mathbf{x}_{3}\right)} d \mathbf{x}_{1} d \mathbf{x}_{2} d \mathbf{x}_{3}
$$

Если в этом уравнении положить $K\left(\mathbf{x}, \mathbf{x}_{1}, \mathbf{x}_{2}, \mathbf{x}_{3}\right)=\delta\left(\mathbf{x}-\mathbf{x}_{1}\right) \delta\left(\mathbf{x}-\mathbf{x}_{2}\right) \delta\left(\mathbf{x}-\mathbf{x}_{3}\right)$, то мы получим широко известное НУШ. В работах [6], [7] строятся квазиклассические решения и изучается распространение гауссовых волновых пакетов в уравнении Шредингера с интегральной нелинейностью типа Хартри, что соответствует уравнению (3) для функции $K\left(\mathbf{x}, \mathbf{x}_{1}, \mathbf{x}_{2}, \mathbf{x}_{3}\right)=K\left(\mathbf{x}, \mathbf{x}_{2}\right) \delta\left(\mathbf{x}-\mathbf{x}_{1}\right) \delta\left(\mathbf{x}_{2}-\mathbf{x}_{3}\right)$.

Выпишем уравнение (3) для ядра $K\left(\mathbf{x}, \mathbf{x}_{1}, \mathbf{x}_{2}, \mathbf{x}_{3}\right)=\delta\left(\mathbf{x}-\mathbf{x}_{1}-\mathbf{x}_{2}+\mathbf{x}_{3}\right)$ и найдем его общее решение:

$$
i \frac{\partial \psi(t, \mathbf{x})}{\partial t}+\Delta \psi(t, \mathbf{x})=\int \delta\left(\mathbf{x}-\mathbf{x}_{1}-\mathbf{x}_{2}+\mathbf{x}_{3}\right) \psi\left(t, \mathbf{x}_{1}\right) \psi\left(t, \mathbf{x}_{2}\right) \overline{\psi\left(t, \mathbf{x}_{3}\right)} d \mathbf{x}_{1} d \mathbf{x}_{2} d \mathbf{x}_{3}
$$


Уравнение (2) здесь имеет простой вид:

$$
i \dot{\psi}(t, \mathbf{p})=\left(\mathbf{p}^{2}+|\psi(t, \mathbf{p})|^{2}\right) \psi(t, \mathbf{p})
$$

Таким образом, общее решение задачи Коши для уравнения (4) с начальным условием $\psi(0, \mathbf{x})$ представляется следующей формулой:

$$
\psi(t, \mathbf{x})=\frac{1}{(2 \pi)^{n}} \int_{R^{n}} \phi(\mathbf{p}) e^{i \mathbf{p} \mathbf{x}-i t\left(\mathbf{p}^{2}+|\phi(\mathbf{p})|^{2}\right)} d \mathbf{p}, \quad \phi(\mathbf{p})=\int_{R^{n}} e^{-i \mathbf{p x}} \psi(0, \mathbf{x}) d \mathbf{x}
$$

Так же, как и в случае линейного уравнения Шредингера, решения уравнения (4) являются суперпозицией плоских волн. Однако наличие в правой части нелинейного члена приводит к существованию качественно новых решений, типичных для нелинейных уравнений, а именно солитонных решений. Под солитонным решением мы понимаем решение в виде бегущей волны с конечной нормой $\int|\psi(t, \mathbf{x})|^{2} d \mathbf{x}<\infty$.

Приведем $N$-солитонное решение уравнения (4) в пространстве $R^{n}$. Пусть постоянный вектор $\mathbf{v}_{j} \in R^{n}$ обозначает скорость $j$-го солитона. Обозначим через $B_{j}$ шар в $R^{n}$ с центром в точке $\mathbf{v}_{j} / 2$ и радиусом $\left|\mathbf{v}_{j}\right| / 2$, тогда $B_{j}=\left\{\mathbf{p} \in R^{n} \mid \mathbf{p} \mathbf{v}_{j}-\mathbf{p}^{2}>0\right\}$. Введем подмножества $M_{j} \subset B_{j}$ такие, что $M_{j} \cap M_{s}=\varnothing$ для любых $j \neq s, j, s=$ $1, \ldots, N$. Обозначим через $\theta_{M_{j}}(\mathbf{p})$ характеристические функции множеств $M_{j}$ :

$$
\theta_{M_{j}}(\mathbf{p})= \begin{cases}1, & \mathbf{p} \in M_{j} \\ 0, & \mathbf{p} \notin M_{j}\end{cases}
$$

Очевидна справедливость равенств

$$
\theta_{M_{j}}^{2}(\mathbf{p})=\theta_{M_{j}}(\mathbf{p}), \quad \theta_{M_{j}}(\mathbf{p}) \theta_{M_{s}}(\mathbf{p})=0, \quad j \neq s
$$

Построим функцию

$$
\phi(\mathbf{p})=\sum_{j=1}^{N} \theta_{M_{j}}(\mathbf{p}) \sqrt{\mathbf{p} \mathbf{v}_{j}-\mathbf{p}^{2}} e^{i \varphi_{j}(\mathbf{p})}
$$

где $\varphi_{j}-$ произвольные вещественные функции. В силу равенств (6) имеем

$$
|\phi(\mathbf{p})|^{2}=\sum_{j=1}^{N} \theta_{M_{j}}(\mathbf{p})\left(\mathbf{p} \mathbf{v}_{j}-\mathbf{p}^{2}\right)
$$

Для произвольных функций $F(\mathbf{p}), f_{s}(\mathbf{p})$ в силу этих же формул справедливо равенство

$$
\int_{R^{n}} F(\mathbf{p}) \theta_{M_{j}}(\mathbf{p}) e^{\sum_{s} f_{s}(\mathbf{p}) \theta_{M_{s}}(\mathbf{p})} d \mathbf{p}=\int_{M_{j}} F(\mathbf{p}) e^{f_{j}(\mathbf{p})} d \mathbf{p}
$$



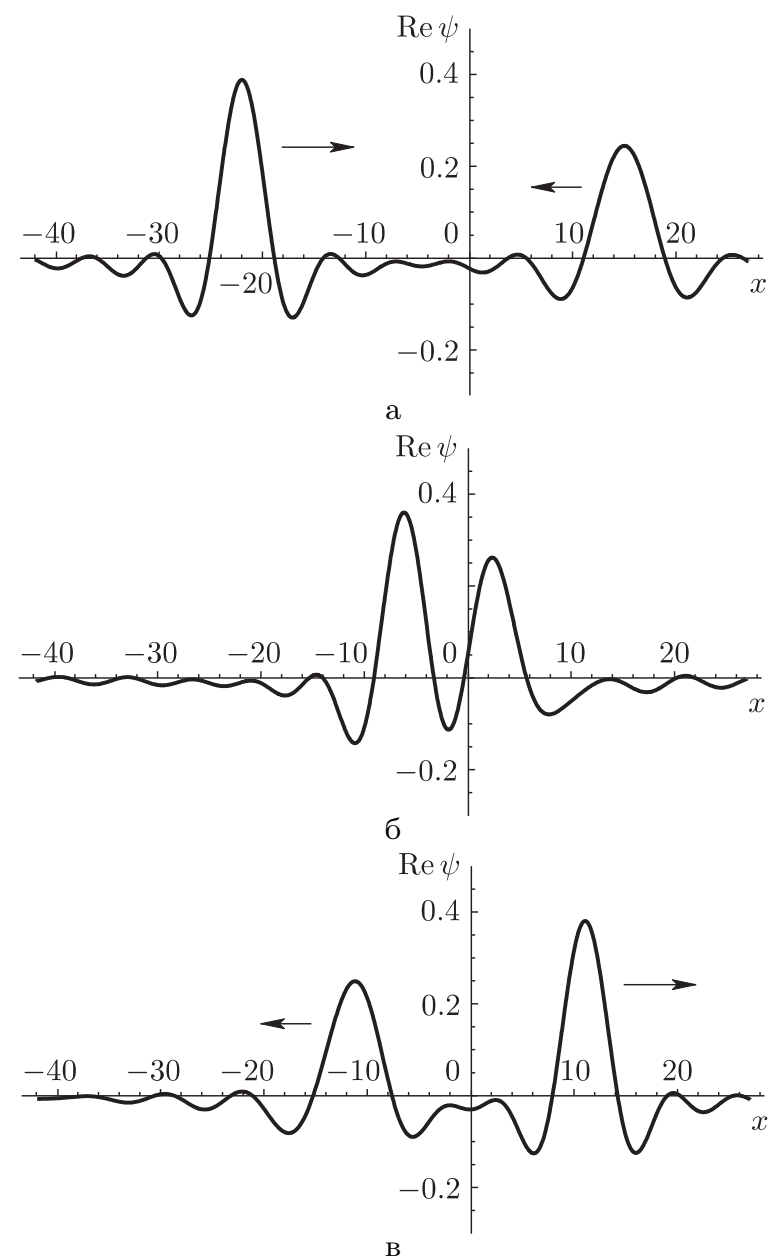

Рис. 1. Двухсолитонное решение в моменты времени $t_{1}=0$ (a), $t_{2}=16$ (б), $t_{3}=33$ (в) для параметров $v_{1}=1, v_{2}=-0.8, x_{1}=-22, x_{2}=15$.

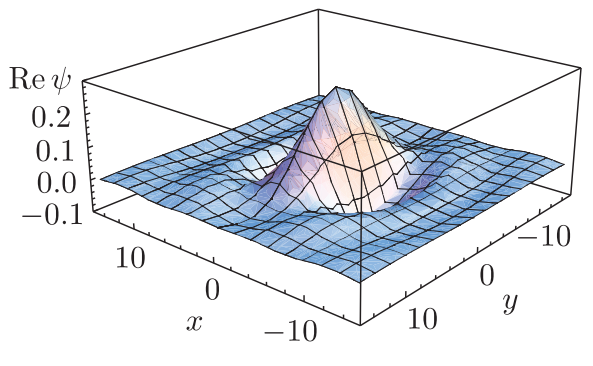

a

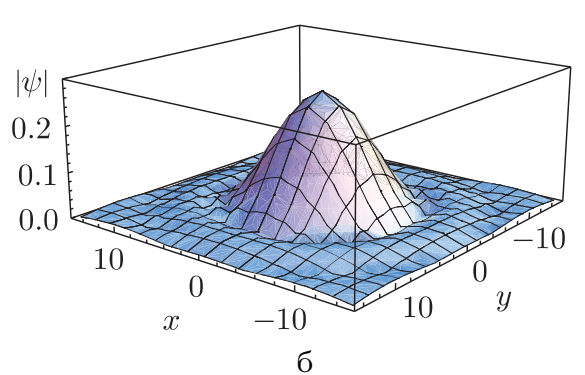

Рис. 2. Односолитонное решение на плоскости для параметров $v_{x}=1, v_{y}=0$. 
Подставляя выражение (7) в формулу общего решения (5) и используя равенства (8) и (9), получаем искомое $N$-солитонное решение

$$
\psi(t, \mathbf{x})=\frac{1}{(2 \pi)^{n}} \sum_{j=1}^{N} \int_{M_{j}} \sqrt{\mathbf{p v}_{j}-\mathbf{p}^{2}} e^{i \varphi_{j}(\mathbf{p})} e^{i \mathbf{p}\left(\mathbf{x}-\mathbf{v}_{j} t\right)} d \mathbf{p} .
$$

Из ограниченности множеств $M_{j}$ и конечности их числа следует конечность нормы солитонного решения (10):

$$
\begin{aligned}
\|\psi\|^{2} & =\int|\psi(t, \mathbf{x})|^{2} d \mathbf{x}=\frac{1}{(2 \pi)^{n}} \sum_{j=1}^{N} \int_{M_{j}}\left(\mathbf{p v}_{j}-\mathbf{p}^{2}\right) d \mathbf{p}< \\
& <\frac{1}{(2 \pi)^{n}} \sum_{j=1}^{N} \mathbf{v}_{j}^{2} \operatorname{Vol}\left(M_{j}\right)<\infty
\end{aligned}
$$

Приведем явный вид решения (10) на $R^{1}$ для случая двух солитонов, движущихся навстречу друг другу со скоростями $v_{1}>0$ и $v_{2}<0$ :

$$
\begin{aligned}
\psi(t, x)= & \frac{v_{1}}{4\left|x-x_{1}-t v_{1}\right|} J_{1}\left(\frac{v_{1}\left|x-x_{1}-t v_{1}\right|}{2}\right) e^{i v_{1}\left(x-x_{1}-t v_{1}\right) / 2}- \\
& -\frac{v_{2}}{4\left|x-x_{2}-t v_{2}\right|} J_{1}\left(-\frac{v_{2}\left|x-x_{2}-t v_{2}\right|}{2}\right) e^{i v_{2}\left(x-x_{2}-t v_{2}\right) / 2},
\end{aligned}
$$

где $J_{1}(z)$ - функции Бесселя первого рода, $x_{1}, x_{2}$ - координаты центра первого и второго солитонов в начальный момент времени. Представление (12) получено из формулы (10) для $M_{1}=B_{1}=\left(0, v_{1}\right), M_{2}=B_{2}=\left(v_{2}, 0\right), \varphi_{1}=-p x_{1}, \varphi_{2}=-p x_{2}$. На рис. 1 представлена вещественная часть решения (12) для трех различных моментов времени.

Приведем также вид двумерного солитона на плоскости $R^{2}$ с координатами $x, y$. На рис. 2 приведены графики вещественной части и модуля волновой функции движущегося вдоль оси $x$ солитона (движение происходит, естественно, без изменения формы).

\section{3. ЭЛЕМЕНТЫ ГАРМОНИЧЕСКОГО АНАЛИЗА НА ГРУППАХ ЛИ}

Если в уравнении (1) считать, что неизвестная функция $\psi$ - функция на абелевой унимодулярной группе Ли $G$, и заменить операторы дифференцирования $-i \partial_{x}$ на действие правоинвариантных векторных полей $\eta$, то мы получим более широкий класс интегрируемых нелинейных интегродифференциальных уравнений:

$$
\begin{aligned}
H\left(\partial_{t}, \eta\right) \psi(t, g)= & A\left(\partial_{t}, \eta\right) \int_{G^{3}} \psi\left(t, g_{1}\right) \psi\left(t, g_{2}\right) \overline{\psi\left(t, g_{3}\right)} \times \\
& \times \delta\left(g g_{3} g_{2}^{-1} g_{1}^{-1}\right) d \mu\left(g_{1}\right) d \mu\left(g_{2}\right) d \mu\left(g_{3}\right)+ \\
& +B\left(\partial_{t}, \eta\right) \int_{G^{5}} \psi\left(t, g_{1}\right) \psi\left(t, g_{2}\right) \psi\left(t, g_{3}\right) \overline{\psi\left(t, g_{4}\right)} \overline{\psi\left(t, g_{5}\right)} \times \\
& \times \delta\left(g g_{5} g_{4} g_{3}^{-1} g_{2}^{-1} g_{1}^{-1}\right) \prod_{k=1}^{5} d \mu\left(g_{k}\right)+\cdots
\end{aligned}
$$


В частном случае, когда оператор $H$ является квадратичной невырожденной комбинацией правоинвариантных полей, мы имеем уравнение скалярного поля с нелокальным самодействием на римановом многообразии группы Ли с правоинвариантной метрикой:

$$
\left(-\frac{\partial^{2}}{\partial t^{2}}+\Delta_{G}-m^{2}\right) \psi(g)=\int_{G^{3}} \psi\left(g_{1}\right) \psi\left(g_{2}\right) \overline{\psi\left(g_{3}\right)} \delta\left(g g_{3} g_{2}^{-1} g_{1}^{-1}\right) d \mu\left(g_{1}\right) d \mu\left(g_{2}\right) d \mu\left(g_{3}\right),
$$

где $\Delta_{G}=g^{a b} \eta_{a} \eta_{b}$, det $g^{a b} \neq 0, g^{a b}=$ const.

В настоящем разделе мы коротко изложим теорию гармонического анализа на группах Ли, основанного на методе орбит коприсоединенного представления, с помощью которой строится метод интегрирования уравнений (13) и (14).

Пусть $G$ - вещественная связная унимодулярная группа Ли, $\mathfrak{g}$ - ее алгебра Ли, $\mathfrak{g}^{*}$ - дуальное пространство к алгебре $\mathfrak{g}, \mathcal{O}_{\lambda} \approx \mathfrak{g}^{*} / G^{\lambda}, G^{\lambda}=\left\{g \in G \mid \operatorname{Ad}_{g}^{*} \lambda=\lambda\right\},-$ орбита коприсоединенного представления группы $G$, проходящая через линейный функционал $\lambda \in \mathfrak{g}^{*}, \omega_{\lambda}-$ симплектическая форма Кириллова:

$$
\omega_{\lambda}(a, b) \equiv\langle\lambda,[X, Y]\rangle, \quad a, b \in T_{\lambda} \mathcal{O}_{\lambda}, \quad X, Y \in \mathfrak{g}, \quad a=\operatorname{ad}_{X}^{*} \lambda, \quad b=\operatorname{ad}_{Y}^{*} \lambda .
$$

Подалгебра $\mathfrak{n} \subset \mathfrak{g}_{\mathbb{C}}$ такая, что

$$
\operatorname{dim} \mathfrak{n}=\operatorname{dim} \mathfrak{g}-\frac{1}{2} \operatorname{dim} \mathcal{O}_{\lambda}, \quad\langle\lambda,[\mathfrak{n}, \mathfrak{n}]\rangle=0,
$$

называется поляризацией линейного функционала $\lambda$. В монографии [8] установлено, что для произвольной алгебры Ли поляризация существует для всякого невырожденного функционала $\lambda$, т.е. когда $\mathcal{O}_{\lambda}$ - орбита общего положения. Отметим, что для гармонического анализа следует рассматривать именно невырожденные функционалы, и в дальнейшем под $\lambda$ мы будем понимать функционал общего положения. В этом случае

$$
\operatorname{dim} \mathcal{O}_{\lambda}=\operatorname{dim} \mathfrak{g}-\text { ind } \mathfrak{g}, \quad \operatorname{dim} \mathfrak{n}=\frac{1}{2}(\operatorname{dim} \mathfrak{g}+\operatorname{ind} \mathfrak{g}), \quad \text { ind } \mathfrak{g} \equiv \inf _{\lambda \in \mathfrak{g}^{*}} \operatorname{dim} \mathfrak{g}^{\lambda} .
$$

По заданной комплексной поляризации $\mathfrak{n} \subset \mathfrak{g}_{\mathbb{C}}$ построим вещественное подпространство $\mathfrak{m}$, так что $\mathfrak{m}_{\mathbb{C}}=\mathfrak{n}+\overline{\mathfrak{n}}$, и вещественную подалгебру $\mathfrak{h}=\mathfrak{n} \cap \overline{\mathfrak{n}} \subset \mathfrak{g}$. Локально всегда выполняется условие

$$
\operatorname{Ad}_{h} \mathfrak{m}=\mathfrak{m}, \quad h \in H .
$$

Обозначим через $\xi_{X}(g)=\left(L_{g}\right)_{*} X, \eta_{X}(g)=-\left(R_{g}\right)_{*} X, X \in \mathfrak{g}$, левоинвариантные и правоинвариантные поля на группе $G$ (генераторы правых и левых сдвигов соответственно). Представление $X \rightarrow \eta_{X}$ алгебры $\mathfrak{g}$ может быть продолжено по линейности до представления комплексной оболочки $\mathfrak{g}_{\mathbb{C}}$. Подалгебра $\mathfrak{n} \subset \mathfrak{g}_{\mathbb{C}}$ подчинена функционалу $\lambda \in \mathfrak{g}_{\mathbb{C}}^{*}$, и поэтому линейный функционал $\lambda$ задает ее одномерное представление $X \rightarrow \lambda(X), X \in \mathfrak{n}$. Определим линейное пространство $L(G, \mathfrak{n}, \lambda)$ решений системы

$$
\left(\eta_{Y}(g)+\frac{i}{\hbar} \lambda(Y)\right) \psi(g)=0, \quad Y \in \mathfrak{n}, \quad \psi \in C^{\infty}(G),
$$

где $\hbar$ - положительный вещественный параметр.

2 Теоретическая и математическая физика, т. 161, № 3, 2009 г. 
Для случая, когда $\mathfrak{m}$ - подалгебра, $H, M$ - замкнутые подгруппы группы $G$ с алгебрами $\mathfrak{h}, \mathfrak{m}$ и выполнено условие (16), в монографии [2] доказывается теорема, которая утверждает, что пространство $L(G, \mathfrak{n}, \lambda)$ изоморфно, т.е. $\phi: L(G, \mathfrak{n}, \lambda) \rightarrow$ $L(Q, \mathfrak{n}, \lambda)$, пространству функций $L(Q, \mathfrak{n}, \lambda)$ на частично голоморфном многообразии $Q$ типа $(k, l)$, где $k=\operatorname{dim} \mathfrak{g}-\operatorname{dim} \mathfrak{m}, l=(\operatorname{dim} \mathfrak{m}-\operatorname{dim} \mathfrak{h}) / 2$. Отметим, что если приведенные выше условия не выполняются, то изоморфизм и многообразие $Q$ определяются локально. Нетрудно проверить справедливость равенства $\operatorname{dim} Q=$ $(1 / 2) \operatorname{dim} \mathcal{O}_{\lambda}=\operatorname{dim} \mathfrak{g}-\operatorname{dim} \mathfrak{n}$. Для функционала $\lambda$ общего положения $\operatorname{dim} Q=$ $(\operatorname{dim} \mathfrak{g}-\operatorname{ind} \mathfrak{g}) / 2$. Важно отметить, что многообразие $Q$ является лагранжевым подмногообразием к орбите $\mathcal{O}_{\lambda}:\left.\omega_{\lambda}\right|_{Q}=0$.

Поскольку пространство $L(G, \mathfrak{n}, \lambda)$ инвариантно относительно правого сдвига, корректно определены линейные операторы $\zeta_{X}$, действующие в пространстве $L(Q, \mathfrak{n}, \lambda)$ :

$$
\zeta_{X}=\phi \circ \xi_{X} \circ \phi^{-1}, \quad X \in \mathfrak{g} .
$$

Операторы $\zeta_{X}$ образуют неприводимое представление алгебры $\mathfrak{g}:\left[\zeta_{X}, \zeta_{Y}\right]=\zeta_{[X, Y]}$, $X, Y \in \mathfrak{g}$, в пространстве $L(Q, \mathfrak{n}, \lambda)$, которое мы называем $\lambda$-представлением.

В локальных координатах $q$ многообразия $Q$ операторы $\zeta_{X}$ имеют вид

$$
\zeta_{X}=\alpha_{X}^{a}(q) \frac{\partial}{\partial q^{a}}+\frac{i}{\hbar} \chi_{X}(q, \lambda), \quad X \in \mathfrak{g}, \quad \chi_{X}(0, \lambda)=\lambda(X) .
$$

Следовательно, $\lambda$-представление можно определить как операторно неприводимое представление алгебры $\mathfrak{g}$ в пространстве функций на лагранжевом подмногообразии $Q$ орбиты коприсоединенного представления $\mathcal{O}_{\lambda}$.

На частично голоморфном многообразии $Q$ введем меру $d \mu(q)$ и скалярное произведение

$$
\left(\psi_{1}, \psi_{2}\right)_{Q} \equiv \int_{Q} \overline{\psi_{1}(q)} \psi_{2}(q) d \mu(q),
$$

относительно которого операторы $\zeta$ антиэрмитовы: $\zeta^{+}=-\zeta$. Для этого необходимо ввести в операторы $\zeta$ "квантовый сдвиг": $\lambda \rightarrow \lambda+i \hbar \beta$, где

$$
\beta(X)=\frac{1}{2}\left(\operatorname{tr}\left(\operatorname{ad}_{X}\right)-\operatorname{tr}\left(\left.\operatorname{ad}_{X}\right|_{\mathfrak{m}}\right)\right), \quad X \in \mathfrak{g} .
$$

Формула (19) справедлива, если пространство $\mathfrak{m}$ - подалгебра. В силу операторной неприводимости операторы Казимира в $\lambda$-представлении являются операторами умножения на вещественные константы: $K(-i \hbar \zeta)=\kappa(\lambda)$.

Пусть $\left(g^{1}, \ldots, g^{\operatorname{dim} \mathfrak{g}}\right)$ - локальные координаты элемента $g$ из окрестности единицы группы $G,\left\{e_{k}=\left.\left(\partial g / \partial g^{k}\right)\right|_{g=e}\right\}$ - базис алгебры $\mathfrak{g} \cong T_{e} G$. Обозначим через $T^{\lambda}$ поднятие $\lambda$-представления алгебры $\mathfrak{g}$ до локального представления группы $G$ :

$$
\left.\frac{\partial T^{\lambda}(g)}{\partial g^{k}}\right|_{g=e}=\zeta_{k} .
$$

По определению

$$
T^{\lambda}(g) \varphi(q)=\int D_{q \bar{q}^{\prime}}^{\lambda}(g) \varphi\left(q^{\prime}\right) d \mu\left(q^{\prime}\right)
$$


для всех $\varphi \in C^{\infty}(Q)$. Из условия $T^{\lambda}\left(g_{1}\right) T^{\lambda}\left(g_{2}\right)=T^{\lambda}\left(g_{1} g_{2}\right)$ следует соотношение для "матричных элементов" представления $T^{\lambda}$ :

$$
D_{q \bar{q}^{\prime}}^{\lambda}\left(g_{1} g_{2}\right)=\int D_{q \bar{q}^{\prime \prime}}^{\lambda}\left(g_{1}\right) D_{q^{\prime \prime} \bar{q}^{\prime}}^{\lambda}\left(g_{2}\right) d \mu\left(q^{\prime \prime}\right)
$$

Используя соотношения

$$
\left.\frac{\partial(g \tilde{g})}{\partial \tilde{g}^{k}}\right|_{\tilde{g}=e}=\xi_{k}(g),\left.\quad \frac{\partial(\tilde{g} g)}{\partial \tilde{g}^{k}}\right|_{\tilde{g}=e}=-\eta_{k}(g)
$$

и формулы $(20),(21)$, получаем уравнения для обобщенных функций $D_{q \bar{q}^{\prime}}^{\lambda}(g)$ :

$$
\left(\eta_{X}(g)+\zeta_{X}(q, \lambda)\right) D_{q \bar{q}^{\prime}}^{\lambda}(g)=0, \quad\left(\xi_{X}(g)+\overline{\zeta_{X}\left(q^{\prime}, \lambda\right)}\right) D_{q \bar{q}^{\prime}}^{\lambda}(g)=0
$$

В силу равенства $\eta\left(g^{-1}\right)=-\xi(g)$ имеем

$$
D_{q \bar{q}^{\prime}}^{\lambda}\left(g^{-1}\right)=\overline{D_{q^{\prime} \bar{q}}^{\lambda}(g)}
$$

С учетом нормировки $D_{q \bar{q}^{\prime}}^{\lambda}(e)=\delta\left(q, \bar{q}^{\prime}\right)$ функции $D_{q \bar{q}^{\prime}}^{\lambda}(g)$ находятся из уравнений $(23)$ однозначно:

$$
D_{q \bar{q}^{\prime}}^{\lambda}\left(e^{X}\right)=e^{\zeta_{X}(q, \lambda)} \delta\left(q, \bar{q}^{\prime}\right), \quad X \in \mathfrak{g} .
$$

Определяя таким образом “матричные элементы" в окрестности единицы и применяя равенство $(22)$, в силу связности группы $G$ можно распространить локальное представление $T^{\lambda}$ на всю группу. Из условия (23) и требования однозначной определенности функций $D_{q \bar{q}^{\prime}}^{\lambda}(g)$ на группе $G$ следует условие для орбиты $\mathcal{O}_{\lambda}$ :

$$
\frac{1}{2 \pi \hbar} \oint_{\gamma \in H_{1}\left(G^{\lambda}\right)} \sigma_{\lambda}=n_{\gamma} \in \mathbb{Z}
$$

Здесь $G^{\lambda}=\left\{g \in G \mid \operatorname{Ad}_{g}^{*} \lambda=\lambda\right\}$ - стабилизатор функционала $\lambda, H_{1}\left(G^{\lambda}\right)$ - группа одномерных гомологий подгруппы $G^{\lambda}, \sigma_{\lambda}$ - левоинвариантная 1-форма на подгруппе $G^{\lambda}$, соответствующая функционалу $\lambda: \sigma_{\lambda}=L_{g}^{*} \lambda, g \in G^{\lambda}$. Подробнее о выводе условия (25) см. работу [9]. Для односвязной группы $G$ условие (25) равносильно условию Кириллова [2] иелочисленности орбиты $\mathcal{O}_{\lambda}$ :

$$
\frac{1}{2 \pi \hbar} \oint_{\gamma \in H_{2}\left(\mathcal{O}_{\lambda}\right)} \omega_{\lambda}=n_{\gamma} \in \mathbb{Z}
$$

Свойства ортогональности и полноты функций $D_{q \bar{q}^{\prime}}^{\lambda}(g)$,

$$
\begin{gathered}
\int D_{q \bar{q}^{\prime}}^{\lambda}(g) \overline{D_{\tilde{q}}^{\tilde{\tilde{q}} \bar{q}^{\prime}}(g)} d \mu(g)=\delta(\lambda, \tilde{\lambda}) \delta(q, \overline{\tilde{q}}) \delta\left(q^{\prime}, \overline{\tilde{q}}^{\prime}\right), \\
\int D_{q \bar{q}^{\prime}}^{\lambda}(g) \overline{D_{q \bar{q}^{\prime}}^{\lambda}(\tilde{g})} d \mu(\lambda) d \mu(q) d \mu\left(q^{\prime}\right)=\delta\left(g \tilde{g}^{-1}\right),
\end{gathered}
$$

позволяют определить прямое и обратное преобразования Фурье:

$$
\psi_{\lambda}\left(q, \bar{q}^{\prime}\right)=\int D_{q \bar{q}^{\prime}}^{\lambda}(g) \psi(g) d \mu(g),
$$




$$
\psi(g)=\int \overline{D_{q \bar{q}^{\prime}}^{\lambda}(g)} \psi_{\lambda}\left(q, \bar{q}^{\prime}\right) d \mu(\lambda) d \mu(q) d \mu\left(q^{\prime}\right)
$$

Ключевым для дальнейших рассуждений является тот факт, что после преобразования (28) действие правоинвариантных и левоинвариантных полей переходит в действие соответствующих операторов $\lambda$-представления и наоборот:

$$
\begin{aligned}
\left(\psi(g) \Longleftrightarrow \psi_{\lambda}\left(q, \bar{q}^{\prime}\right)\right) & \Longleftrightarrow\left(\eta_{X} \psi(g) \Longleftrightarrow \zeta_{X}(q, \lambda) \psi_{\lambda}\left(q, \bar{q}^{\prime}\right),\right. \\
\xi_{X} \psi(g) & \left.\Longleftrightarrow \overline{\zeta_{X}\left(q^{\prime}, \lambda\right)} \psi_{\lambda}\left(q, \bar{q}^{\prime}\right)\right) .
\end{aligned}
$$

Векторные поля $\eta$ и $\xi$ зависят от $\operatorname{dim} \mathfrak{g}$ переменных, в то время как число переменных, входящих в операторы $\zeta(q, \lambda)$, равно $(\operatorname{dim} \mathfrak{g}-\operatorname{ind} \mathfrak{g}) / 2$. Это позволяет применять обобщенный фурье-анализ для редукции дифференциальных операторов, являющихся линейными или полиномиальными комбинациями правоинвариантных и левоинвариантных полей, к операторам с меньшим числом переменных.

Отметим, что классическое преобразование Фурье является частным случаем преобразования (28), соответствующим абелевой группе $R^{n}$.

\section{4. ИНТЕГРИРОВАНИЕ НЕЛИНЕЙНЫХ ИНТЕГРОДИФФЕРЕНЦИАЛЬНЫХ УРАВНЕНИЙ НА ГРУППАХ ЛИ}

Применим изложенный выше метод обобщенного фурье-анализа для интегрирования уравнений (13) и (14). Для этого представим неизвестную функцию в виде $(29)$ и умножим правую и левую части этих уравнений на функцию $\overline{D_{q \bar{q}^{\prime}}^{\lambda}(g)}$. Используя свойства ортогональности и полноты (26), (27), а также равенства (22), (24), сведем уравнение (13) к следующему интегродифференциальному уравнению:

$$
\begin{aligned}
H\left(\partial_{t},\right. & \zeta(q, \lambda)) \psi_{\lambda}\left(t, q, \bar{q}^{\prime}\right)=A\left(\partial_{t}, \zeta(q, \lambda)\right) \int_{Q \times Q} \psi_{\lambda}(t, q, \bar{r}) \psi_{\lambda}\left(t, r, \bar{r}^{\prime}\right) \times \\
& \times \overline{\psi_{\lambda}\left(t, q^{\prime}, \bar{r}^{\prime}\right)} d \mu(r) d \mu\left(r^{\prime}\right)+ \\
& +B\left(\partial_{t}, \zeta(q, \lambda)\right) \int_{Q^{4}} \psi_{\lambda}(t, q, \bar{r}) \psi_{\lambda}(t, r, \bar{s}) \psi_{\lambda}\left(t, s, \bar{s}^{\prime}\right) \times \\
& \times \overline{\psi_{\lambda}\left(t, r^{\prime}, \bar{s}^{\prime}\right)} \frac{\psi_{\lambda}\left(t, q^{\prime}, \bar{r}^{\prime}\right)}{\psi^{\prime}} d \mu(r) d \mu\left(r^{\prime}\right) d \mu(s) d \mu\left(s^{\prime}\right)+\cdots .
\end{aligned}
$$

Уравнение (30) содержит $\operatorname{dim} \mathfrak{g}$ - ind $\mathfrak{g}$ "пространственных" переменных $q, q^{\prime}$ вместо $\operatorname{dim} \mathfrak{g}$ изначальных переменных $g$. Отметим, что такая же ситуация была бы и в случае, если операторы $H, A, B, \ldots$ в уравнении (13) содержали бы, кроме правоинвариантных полей $\eta$, также и левоинвариантные поля $\xi$. В случае правоинвариантных и самосопряженных в пространстве $L_{2}(G, d \mu(g))$ операторов $H, A, B, \ldots$ можно провести дальнейшую редукцию.

Рассмотрим подробнее уравнение (14). Соответствующее редуцированное уравнение (30) в этом случае имеет вид

$$
\left(-\frac{\partial^{2}}{\partial t^{2}}+\widetilde{\Delta}_{\lambda}-m^{2}\right) \psi_{\lambda}\left(t, q, \bar{q}^{\prime}\right)=\int_{Q \times Q} \psi_{\lambda}(t, q, \bar{r}) \psi_{\lambda}\left(t, r, \bar{r}^{\prime}\right) \overline{\psi_{\lambda}\left(t, q^{\prime}, \bar{r}^{\prime}\right)} d \mu(r) d \mu\left(r^{\prime}\right)
$$


где $\widetilde{\Delta}_{\lambda} \equiv g^{a b} \zeta_{a}(q, \lambda) \zeta_{b}(q, \lambda)$.

Рассмотрим сначала случай, когда группа $G$ компактна. Для компактной группы $G$ представления $T^{\lambda}$ конечномерны и, как следствие, спектр оператора $\widetilde{\Delta}_{\lambda}$ также конечен. В силу косоэрмитовости операторов $\zeta_{a}(q, \lambda)$ в пространстве $L_{2}(Q, d \mu(q))$ оператор $\widetilde{\Delta}_{\lambda}$ самосопряжен, поэтому существует полный ортонормированный набор его собственных функций $\left\{\varphi_{A}(q)\right\}\left(\left(\varphi_{A}, \varphi_{B}\right)_{Q}=\delta_{A B}\right)$ :

$$
-g^{a b} \zeta_{a}(q, \lambda) \zeta_{b}(q, \lambda) \varphi_{A}(q)=\omega_{A}^{2} \varphi_{A}(q)
$$

Разложим функцию $\psi_{\lambda}\left(t, q, \bar{q}^{\prime}\right)$ по этому набору:

$$
\psi_{\lambda}\left(t, q, \bar{q}^{\prime}\right)=\sum_{A, B=1}^{\operatorname{dim} T^{\lambda}} \Psi_{A B}^{\lambda}(t) \varphi_{A}(q) \overline{\varphi_{B}\left(q^{\prime}\right)} .
$$

Подставляя разложение (33) в формулу (32), получаем автономную систему из $\operatorname{dim} T^{\lambda}$ обыкновенных дифференциальных уравнений для матричных элементов матрицы $\Psi^{\lambda}$ :

$$
\left(-\frac{\partial^{2}}{\partial t^{2}}-\omega^{2}-m^{2}\right) \Psi^{\lambda}=\left(\Psi^{\lambda}\right)^{2}\left(\Psi^{\lambda}\right)^{+}
$$

где $\omega^{2}=\operatorname{diag}\left(\omega_{A}^{2}\right)$, знак “+” означает операцию эрмитова сопряжения матрицы.

Таким образом, построение общего решения интегродифференциального уравнения (14) сводится к решению линейной задачи на собственные значения (32) и к интегрированию автономной системы обыкновенных дифференциальных уравнений (34). Отметим, что задача на собственные значения (32) в силу конечномерности пространства представления является чисто алгебраической.

Рассмотрим теперь случай некомпактной группы. Пусть $M_{A}, A=1, \ldots, N_{\lambda},-$ непересекающиеся открытые подмножества многообразия $Q$, инвариантные относительно инволюции комплексного сопряжения: $\bar{M}_{A}=M_{A}, M_{A} \cap M_{B}=\varnothing, A \neq B$. Обозначим через $\theta_{A}(q)$ характеристические функции множеств $M_{A}$ :

$$
\theta_{A}(q)=\left\{\begin{array}{ll}
1, & q \in M_{A}, \\
0, & q \notin M_{A},
\end{array} \quad q \in M_{A} \Leftrightarrow \bar{q} \in M_{A},\right.
$$

которые обладают очевидными свойствами

$$
\overline{\theta_{A}(q)}=\theta_{A}(q), \quad \theta_{A}^{2}(q)=\theta_{A}(q), \quad \theta_{A}(q) \theta_{B}(q)=0, \quad A \neq B
$$

Из бесконечного спектра оператора $\widetilde{\Delta}_{\lambda}$ выберем произвольным образом $N_{\lambda}$ точек $\left\{\omega_{A}\right\}$ и представим функцию $\psi_{\lambda}\left(t, q, \bar{q}^{\prime}\right)$ в виде

$$
\psi_{\lambda}\left(t, q, \bar{q}^{\prime}\right)=\sum_{A, B=1}^{N_{\lambda}} \Psi_{A B}^{\lambda}(t) \theta_{A}(q) \varphi_{A}(q) \overline{\theta_{B}\left(q^{\prime}\right)} \overline{\varphi_{B}\left(q^{\prime}\right)} .
$$

Здесь $\varphi_{A}(q)$ - собственные функции оператора $\widetilde{\Delta}_{\lambda}$, отвечающие собственному значению $\omega_{A}^{2}(32)$, нормированные условием $\int_{M_{A}} \varphi_{A}(q) \overline{\varphi_{A}(q)} d \mu(q)=1$. Подставляя 
разложение (36) в формулу (31), с учетом нормировки функций $\varphi_{A}$ и равенств (35) получаем для матрицы $\Psi^{\lambda}$ уравнение (34).

Таким образом, как в компактном, так и в некомпактном случае интегрирование интегродифференциального уравнения (14) сводится к решению линейной задачи на собственные значения (32) и к интегрированию системы обыкновенных дифференциальных уравнений (34). Разница в том, что в компактном случае, перебирая все представления, мы получаем все решения, в некомпактном же случае, учитывая произвол в выборе семейства $\left\{\omega_{A}^{2}\right\}$ точек спектра и подмножеств $M_{A}$, получаем бесконечное множество частных решений.

ЗАмЕчАниЕ. Несмотря на то что для каждого фиксированного представления $T^{\lambda}$ система (34) конечномерна, иногда "перебрать" все представления даже для компактного случая невозможно. Поэтому в этом случае для построения решений мы выбираем произвольное (может быть, конечное) число представлений и считаем, что интегралы по спектральной мере $\int(\cdot) d \mu(\lambda)$ берутся по выбранным представлениям и являются конечными.

В общем случае представить решение системы (34) в матричном виде не представляется возможным. Однако мы можем предъявить два больших класса решений.

Нетрудно проверить, что матрица $\Psi^{\lambda}=e^{i \Omega t} U$, где $\Omega=\operatorname{diag}\left(\Omega_{A}\right), \Omega_{A}=\sqrt{\omega_{A}^{2}+m^{2}}$, $U$ - произвольная унитарная матрица, является решением системы (34). Для этого решения норма волновой функции не зависит от времени:

$$
\|\psi\|^{2}=\int_{G} \overline{\psi(t, g)} \psi(t, g) d \mu(g)=\int N_{\lambda} d \mu(\lambda)
$$

(в компактном случае $N_{\lambda}=\operatorname{dim} T^{\lambda}$ ).

Ко второму классу решений относятся так называемые СРТ-инвариантные решения, где $\mathrm{C}$ - операция зарядового сопряжения (для скалярного поля это комплексное сопряжение), Р, Т - операции пространственного и временно́го отражения соответственно. Примером СРТ-инвариантного решения является плоская волна $e^{i(\omega t-p x)}$, поэтому можно говорить, что СРТ-инвариантные решения являются обобщениями плоских волн.

Естественным обобщением пространственной инверсии на неабелеву группу является следующая инволюция: $P \psi(g)=\psi\left(g^{-1}\right)$. Таким образом, СРТ-инвариантное решение удовлетворяет условию $\overline{\psi(t, g)}=\psi\left(-t, g^{-1}\right)$. Для матрицы $\Psi^{\lambda}$ в силу унитарности представления (24) это эквивалентно условию $\left(\Psi^{\lambda}\right)^{+}(t)=\Psi^{\lambda}(-t)$. Решением задачи (34), удовлетворяющим данному условию, является диагональная матрица

$$
\Psi^{\lambda}(t)=\operatorname{diag}\left(e^{i \Omega_{A}(\lambda) t} \sqrt{\Omega_{A}^{2}(\lambda)-\omega_{A}^{2}-m^{2}}\right),
$$

где $\Omega_{A}$ - произвольные вещественные числа такие, что $\Omega_{A}^{2}-\omega_{A}^{2}-m^{2} \geqslant 0$. Подставляя матрицу (37) в формулу (36) (или (33) в компактном случае), а затем результат в формулу (29), получаем СРТ-инвариантное решение

$$
\psi(t, g)=\int_{\widehat{G}} \sum_{A} \sqrt{\Omega_{A}^{2}-\omega_{A}^{2}-m^{2}} e^{i \Omega_{A}(\lambda) t} \chi_{A}^{\lambda}(g) d \mu(\lambda),
$$


где

$$
\chi_{A}^{\lambda}(g)=\int_{M_{A} \times M_{A}} \overline{D_{q \bar{q}^{\prime}}^{\lambda}}(g) \varphi_{A}(q) \overline{\varphi_{A}\left(q^{\prime}\right)} d \mu(q) d \mu\left(q^{\prime}\right) .
$$

Для компактной группы в формуле (39) следует положить $M_{A}=Q$. Решение (38) можно обобщить, введя произвольные "фазы” $\alpha_{A}(\lambda)$ :

$$
\psi(t, g)=\int_{\widehat{G}} \sum_{A} \sqrt{\Omega_{A}^{2}-\omega_{A}^{2}-m^{2}} e^{i \Omega_{A}(\lambda) t} e^{i \alpha_{A}(\lambda)} \chi_{A}^{\lambda}(g) d \mu(\lambda) .
$$

Функции $\chi_{A}^{\lambda}(g)$ обладают следующими важными свойствами:

$$
-\Delta_{G} \chi_{A}^{\lambda}(g)=\omega_{A}^{2} \chi_{A}^{\lambda}(g), \quad \int \overline{\chi_{A}^{\lambda}(g)} \chi_{B}^{\tilde{\lambda}}(g) d \mu(g)=\delta(\lambda, \tilde{\lambda}) \delta_{A B}, \quad \overline{\chi_{A}^{\lambda}(g)}=\chi_{A}^{\lambda}\left(g^{-1}\right) .
$$

В компактном случае $\sum_{A=1}^{\operatorname{dim} T^{\lambda}} \chi_{A}^{\lambda}(g)=\chi^{\lambda}(g)$ - характер представления $T^{\lambda}$. С учетом этих свойств решение (40) по форме напоминает $N$-солитонное решение $(10)$. Решение (40) также обладает конечной нормой

$$
\|\psi\|^{2}=\int_{G} \overline{\psi(t, g)} \psi(t, g) d \mu(g)=\int \sum_{A}\left(\Omega_{A}^{\lambda}-\omega_{A}^{2}-m^{2}\right) d \mu(\lambda)
$$

(интересно сравнить эту формулу с формулой (11)). В разделе 5 мы приведем решение (40) для группы $S O(3)$.

\section{5. УРАВНЕНИЕ НА ГРУППЕ $S O(3)$}

Группа вращений трехмерного евклидова пространства $S O(3)$ представляет собой трехмерную вещественную компактную группу Ли. Пусть $x^{1}, x^{2}, x^{3}$ - канонические координаты второго рода элемента $g \in S O(3): g=e^{x^{3} e_{3}} e^{x^{2} e_{2}} e^{x^{1} e_{1}}$, где $e_{i}$ - базис алгебры $s o(3), x^{1}, x^{3} \in[0 ; 2 \pi), x^{2} \in[-\pi / 2 ; \pi / 2)$. Они имеют смысл углов вращения вокруг трех взаимно перпендикулярных осей. Выпишем инвариантную меру и правоинвариантные поля в этих координатах:

$$
\begin{gathered}
d \mu(g)=\frac{1}{8 \pi} \cos x^{2} d x^{1} d x^{2} d x^{3}, \quad \eta_{1}=-\frac{\cos x^{3}}{\cos x^{2}} \partial_{x^{1}}+\sin x^{3} \partial_{x^{2}}-\operatorname{tg} x^{2} \cos x^{3} \partial_{x^{3}}, \\
\eta_{2}=-\frac{\sin x^{3}}{\cos x^{2}} \partial_{x^{1}}+\cos x^{3} \partial_{x^{2}}-\operatorname{tg} x^{2} \sin x^{3} \partial_{x^{3}}, \quad \eta_{3}=-\partial_{x^{3}} .
\end{gathered}
$$

Каждая целочисленная орбита коприсоединенного представления проходит через ковектор $\lambda=(j, 0,0), j=0,1,2, \ldots$ Форма Кириллова на целочисленных К-орбитах $\mathcal{O}_{\lambda}=\left\{f \in s o(3)^{*} \mid K(f)=f_{1}^{2}+f_{2}^{2}+f_{3}^{2}=j^{2}, j=0,1,2, \ldots\right\}$ имеет вид $\omega_{\lambda}=$ $d f_{1} \wedge d f_{2} / f_{3}$. Выпишем $\lambda$-представление, соответствующее поляризации $\left\{e_{1}, e_{2}+i e_{3}\right\}$ (полагаем $\hbar=1)$ :

$$
\zeta_{1}=-i \sin q \partial_{q}+i j \cos q, \quad \zeta_{2}=-i \cos q \partial_{q}-i j \sin q, \quad \zeta_{3}=\partial_{q} .
$$

Здесь $q=\alpha+i \beta, \alpha \in[0 ; 2 \pi), \beta \in(-\infty ; \infty)$. Операторы $\zeta_{i}$ косоэрмитовы относительно меры

$$
d \mu_{j}(q)=\frac{(2 j+1) ! ! d \alpha d \beta}{\pi 2^{j+2} j !(\operatorname{ch} \beta)^{2 j+2}} .
$$


Спектральная мера оператора Казимира $K(i \eta)$ дается выражением

$$
\int(\cdot) d \mu(\lambda)=\sum_{j=0}^{\infty}(\cdot)(2 j+1) .
$$

"Матричные элементы" представления $T^{\lambda}$ имеют вид

$$
\begin{aligned}
D_{q \bar{q}^{\prime}}^{j}(g)= & e^{x^{3} \zeta_{3}(q, j)} e^{x^{2} \zeta_{2}(q, j)} e^{x^{1} \zeta_{1}(q, j)} \delta\left(q, \bar{q}^{\prime}\right)= \\
= & \frac{2^{j}(j !)^{2}}{(2 j) !}\left[\left(\cos x^{1} \cos \bar{q}^{\prime}+i \sin x^{1}\right) \cos \left(q+x^{3}\right)+\right. \\
& \quad+\left(\sin x^{1} \sin x^{2} \cos \bar{q}^{\prime}-\cos x^{2} \sin \bar{q}^{\prime}-i \cos x^{1} \sin x^{2}\right) \sin \left(q+x^{3}\right)+ \\
& \left.\quad+i \sin x^{2} \sin \bar{q}^{\prime}+\cos x^{1} \cos x^{2}+i \sin x^{1} \cos x^{2} \cos \bar{q}^{\prime}\right]^{j}
\end{aligned}
$$

Рассмотрим уравнение (14) на группе $S O(3)$. В качестве оператора $\Delta_{S O(3)}$ выберем "возмущенный" оператор Казимира

$$
\Delta_{S O(3)}=K(\eta)+\varepsilon \eta_{3}^{2}=\eta_{1}^{2}+\eta_{2}^{2}+(1+\varepsilon) \eta_{3}^{2}, \quad \varepsilon>0 .
$$

После редукции он переходит в оператор

$$
\widetilde{\Delta}_{\lambda}=K(\zeta(q, j))+\varepsilon \zeta_{3}(q, j)^{2}=-j(j+1)+\varepsilon \partial_{q}^{2} .
$$

Нормированные собственные функции оператора $-\widetilde{\Delta}_{\lambda}$, соответствующие собственным значениям $\omega_{A}^{2}=\varepsilon A^{2}+j(j+1)$, равны

$$
\varphi_{A}(q)=\frac{j ! e^{i A q}}{\sqrt{(j+A) !(j-A) !}}, \quad A=-j,-j+1, \ldots, j .
$$

Найдем функции $\chi_{A}^{\lambda}(g)$. Используя определение (39) и равенство (41), получаем

$$
\begin{aligned}
\chi_{A}^{j}(g)= & \int \varphi_{A}(q) \overline{\varphi_{A}\left(q^{\prime}\right)} e^{x^{3} \overline{\zeta_{3}(q, j)}} e^{x^{2} \overline{\zeta_{2}(q, j)}} e^{x^{1} \overline{\zeta_{1}(q, j)}} \overline{\delta\left(q, \bar{q}^{\prime}\right)} d \mu_{j}(q) d \mu_{j}\left(q^{\prime}\right)= \\
= & e^{-i A x^{3}} \int \overline{e^{x^{1} \zeta_{1}(q, j)} \varphi_{A}(q)} e^{-x^{2} \zeta_{2}(q, j)} \varphi_{A}(q) d \mu_{j}(q)= \\
= & \frac{j !^{2} e^{-i A x^{3}}}{(j+A) !(j-A) !} \int\left(\cos x^{1} \cos \bar{q}-i\left(\sin x^{1}+\sin \bar{q}\right)\right)^{A} \times \\
& \times\left(\cos x^{1}-i \sin x^{1} \cos \bar{q}\right)^{j-A} \times \\
& \times\left(\cos q-\sin x^{2}+i \cos x^{2} \sin q\right)^{A}\left(\cos x^{2}+i \sin x^{2} \sin q\right)^{j-A} d \mu_{j}(q) .
\end{aligned}
$$

Построим СРТ-инвариантное решение (40) для простейшего случая $\Omega_{A}^{2}(j)=$ $\omega_{A}(j)^{2}+m^{2}$, где параметр $j$ принимает два значения $j=0$ и $j=1$ :

$$
\begin{aligned}
\psi(t, g) & =\sqrt{\Omega^{2}-m^{2}} e^{i \Omega t} \chi_{0}^{0}(g)+3 \sum_{A=-1}^{1} \sqrt{\Omega_{A}^{2}-\varepsilon A^{2}-2-m^{2}} e^{i \Omega_{A} t} \chi_{A}^{1}(g)= \\
& =\sqrt{\Omega^{2}-m^{2}} e^{i \Omega t}+\frac{3}{2}\left(\sqrt{\Omega_{-1}^{2}-\varepsilon-2-m^{2}} e^{i\left(\Omega_{-1} t+x^{3}\right)} \times\right.
\end{aligned}
$$




$$
\begin{aligned}
& \times\left(\cos x^{1}+\cos x^{2}-i \sin x^{1} \sin x^{2}\right)+ \\
& +2 \sqrt{\Omega_{0}^{2}-2-m^{2}} e^{i \Omega_{0} t} \cos x^{1} \cos x^{2}+ \\
& \left.+\sqrt{\Omega_{1}^{2}-\varepsilon-2-m^{2}} e^{i\left(\Omega_{1} t-x^{3}\right)}\left(\cos x^{1}+\cos x^{2}+i \sin x^{1} \sin x^{2}\right)\right) .
\end{aligned}
$$

Здесь введены обозначения $\Omega=\Omega_{0}(0), \Omega_{A}=\Omega_{A}(1)$. Заметим, что первое и третье слагаемые в скобках представляют собой “волновые решения", т.е. решения, удовлетворяющие условию $\psi(t+a, g)=\psi\left(t, h^{-1}(a) g\right)$ с $h(a)=e^{a \Omega_{ \pm 1} e_{3}}$.

\section{6. ЗАКЛЮЧЕНИЕ}

Можно показать, что уравнение (4), как и классические нелинейные уравнения, интегрируемые методом обратной задачи рассеяния, представляет собой бесконечномерную гамильтонову систему, а преобразование Фурье играет для него роль перехода к переменным типа действие-угол. Интегрируемость уравнения (4) обеспечивается наличием у него бесконечнопараметрической группы симметрий. В связи с этим представляет интерес исследование симметрий уравнений более общего вида (13).

\section{Список литературы}

[1] В. Е. Захаров, С. В. Манаков, С. П. Новиков, Л. П. Питаевский, Теория солитонов: Метод обратной задачи, Наука, М., 1980.

[2] А.А. Кириллов, Элементы теории представлений, Наука, М., 1978.

[3] И. В. Широков, К-орбиты, гармонический анализ на однородных пространствах и интегрирование дифференциалъных уравнений, ОмГУ, Омск, 1998.

[4] А. В. Шаповалов, И. В. Широков, ТМФ, 104:2 (1995), 195-213.

[5] С. П. Барановский, В. В. Михеев, И. В. Широков, ТМФ, 129:1 (2001), 3-13.

[6] В. В. Белов, А. Ю. Трифонов, А. В. Шаповалов, ТМФ, 130:3 (2002), 460-492.

[7] Й. Брюнинг, С. Ю. Доброхотов, Р. В. Некрасов, А. И. Шафаревич, ТМФ, 155:2 (2008), $215-235$.

[8] Ж. Диксмье, Универсальные обертывающие алгебры, Мир, М., 1978.

[9] И. В. Широков, ТМФ, 123:3 (2000), 407-423. 